\title{
Quantitative genetics of female choice in an ultrasonic pyralid moth, Achroia grisella: variation and evolvability of preference along multiple dimensions of the male advertisement signal
}

\author{
YIKWEON JANG* \& MICHAEL D. GREENFIELD \\ Department of Entomology, University of Kansas, Lawrence, KS 66045, U.S.A.
}

\begin{abstract}
The mating system of Achroia grisella (Lepidoptera: Pyralidae) is characterized by male ultrasonic advertisement signalling to which females orientate. Although males provide no direct, somatic benefits to their mates, females prefer males whose signal characters are more exaggerated than the population means. Previous studies showed that the signal characters influencing mate attraction are highly repeatable and heritable. We measured the phenotypic and additive genetic variances (heritability) of female preference in A. grisella, as this additive genetic variance is one of the genetic assumptions of indirect models of sexual selection. We determined the preference index of female A. grisella by repeated phonotaxis trials in which a choice of simulated male signals was presented. These playback experiments showed that female preference indices varied but were repeatable within individuals. Specifically, females differ in the relative importance of the several signal characters during mate assessment. A subsequent half-sib breeding design revealed an amount of additive genetic variance for the female preference index $\left(h_{\mathrm{s}}^{2}=0.212, \mathrm{SE}=0.1347, P=0.0611 ; C V_{\mathrm{A}}=0.1826\right)$. Our study highlights the importance of careful preparation of test signals and experimental design for quantifying individual variation in (female) preference along multiple signal dimensions.
\end{abstract}

Keywords: acoustic communication, female preference, indirect sexual selection.

\section{Introduction}

The processes of sexual selection include competition within one sex, generally the male, for access to the other sex and intersexual selection in which one sex, generally the female, mates preferentially with certain members of the other sex based on various criteria. These criteria may be somatic, but in many species females discriminate among potential mates even when they accrue little or no direct, somatic benefit by virtue of mating with one male over another. In such cases it is proposed that females receive indirect, genetic benefits in the form of 'attractiveness alleles' (arbitrary, or Fisherian, model: Lande, 1981; Kirkpatrick, 1982) and 'viability alleles' (good-genes model: Pomiankowski, 1988) inherited by their offspring.

\footnotetext{
*Correspondence and present address: Y. Jang, Center for the Integrative Study of Animal Behaviour, Indiana University, Bloomington, IN 47405, U.S.A. E-mail: yjang@indiana.edu
}

Both the arbitrary and good-genes models of sexual selection are coevolutionary in that the male character and female preference coevolve in a reciprocally reinforcing manner. These models assume the existence of: (i) significant additive genetic variance (heritability) for both the male character and (ii) the female preference, as well as (iii) genetic correlation between these male and female traits (Kirkpatrick \& Ryan, 1991). This genetic correlation may arise from the linkage disequilibrium established by assortative mating, and it may lead to extreme exaggeration of the male character and female preference. Confirmation of these three fundamental assumptions and determination that selection is occurring does not indicate which indirect model of sexual selection is operating, but failure to demonstrate the assumptions would suggest that neither model is responsible for the observed selection. Rather, a direct mechanism such as exploitation of sensory bias would be implicated. 
Whereas various workers have reported evidence for heritability of male signal characters in diverse species, only a few have reported parallel findings for female preference. This disparity may reflect the relative difficulty of studying behavioural preference traits as opposed to morphological and signalling ones (Jang, 1997) or the lack of attention to and thorough analyses of preference in sexual selection studies (Rosenqvist \& Berglund, 1992; Jennions \& Petrie, 1997).

Several investigators have conducted genetic analyses of mate preference in species wherein different females exhibit markedly distinct choices (for review, see Bakker \& Pomiankowski, 1995). Their studies have revealed genetic control of preferences by relatively few loci. But preferences in most species are continuous rather than discrete, indicating that they are polygenic and should be examined via quantitative genetic analyses of intrapopulation variation. A limited number of quantitative genetic studies have reported correlated responses of preference to artificial selection on male characters that suggested some genetic influence on female choice (for review, see Bakker \& Pomiankowski, 1995; Jennions \& Petrie, 1997). Whereas these correlated responses may suffice to confirm the fundamental genetic assumptions of indirect sexual selection models, they do not provide specific estimates of additive genetic variance, and heritability, of choice. Such estimates would be necessary to predict quantitatively a population's response to indirect sexual selection.

Here, we report a study examining phenotypic and additive genetic variation in female preference in the lesser wax moth Achroia grisella (Lepidoptera: Pyralidae). We exploited the relative ease with which female A. grisella can be tested and bred in the laboratory to calculate reliable preference indices for each individual and to determine the heritability of the index.

In the field $A$. grisella is a symbiont of honeybees (Apis mellifera). Unlike most Lepidoptera, pair formation in A. grisella entails ultrasonic advertisement signals by stationary, wing-fanning males that attract receptive females within a radius of several metres (Spangler et al., 1984). Experiments using simulated signals showed that the ultrasound alone was as attractive as a live male. Acoustic signals in male A. grisella are produced by a pair of tymbals on the tegulae (see Spangler et al., 1984). The tegulae are struck twice by the bases of the forewings during each complete cycle of wing movement ( $=1$ period), once on the upstroke and once on the downstroke. A 100- $\mu$ s pulse of sound is emitted from the tymbal, which buckles in or out as the tegula is struck. Owing to slightly asynchronous wing movement, the insect's two tymbals produce a pair of pulses during each upstroke and downstroke that are usually separated by a brief $(100-400 \mu \mathrm{s})$ silent gap. The sound pulses are loud [sound pressure level $(\mathrm{SPL})=95 \mathrm{~dB}$ at $1 \mathrm{~cm} ; 0 \mathrm{~dB}$ re $20 \mu \mathrm{Pa}$ ] and include frequencies from 70 to $130 \mathrm{kHz}$. There exists no evidence that direct, somatic benefits of any kind, e.g. parental care or spermatophore nutrients, are provided by males and influence female preference (Greenfield \& Coffelt, 1983).

Playback experiments and multivariate regression analyses revealed that the signal characters, pulse rate (PR), asynchrony interval (AI) and peak amplitude (PA), are the targets of female choice in A. grisella (Jang \& Greenfield, 1996, 1998). Pulse rate is a measure of signal repetition rate and is defined as the inverse of the period between the beginning of a given pair of pulses to the beginning of the following pair of pulses. Asynchrony interval is the time interval from the beginning of the first pulse to the beginning of the second pulse in a pair of pulses produced by a given upstroke or downstroke. Peak amplitude is calculated as the greatest absolute value of the SPLs sampled during a pulse. On average, females prefer signals with PRs, AIs and PAs greater than mean values in the male population.

The three male signal characters influencing female preference vary considerably within populations but are repeatable within individuals (Jang et al., 1997). Quantitative genetic experiments using a half-sib design determined that heritabilities $\left(h_{\mathrm{s}}^{2}\right)$ of all three signal characters are significant among collectively reared moths, but only $h_{\mathrm{s}}^{2}$ of PA is significant in individually reared moths (Collins et al., 1999). Estimates of phenotypic and genetic variance, including 'evolvability' (sensu Houle, 1992), of female preference would complement the above information and further our understanding of the potential for sexual selection to operate in A. grisella.

\section{Materials and methods}

\section{Population studied}

We studied a laboratory population of A. grisella derived from animals collected at infested honeybee colonies near Auburn, Alabama during September 1994. Throughout the 16 generations between founding and the study reported here, we maintained a random breeding protocol that minimized loss of genetic variation in the stock population. Larvae were reared on a diet containing a flour mixture, glycerine, Brewer's yeast, beeswax, honey and water (Dutky et al., 1962) and kept under a 12:12 h light:dark photoperiod at $25.5^{\circ} \mathrm{C}$. To ensure that the condition of tested adults was standardized, we used virgin and individually reared animals for all experiments. Virgin adults were obtained by individually placing second-instar larvae from the 
stock population in 30-mL containers supplied with diet ad libitum $(\approx 1 \mathrm{~g})$. These larvae pupated and eclosed to adults within the individual containers.

\section{Measurement of female preference index}

Because an acoustic signal of a male A. grisella is defined by several characters and male attractiveness is a function of these signal characters, female preference functions can be projected to multidimensional signal coordinates (Jang \& Greenfield, 1998). However, such multidimensionality would render the characterization of preference functions of individual females over the entire range of signal coordinates rather complex. Therefore, we determined preference indices of individual females by using two-stimulus choice tests especially designed to reveal any fundamental differences in the relative importance of the various signal characters during mate assessment.

To measure the preference indices of individual females accurately, we repeatedly tested their phonotaxis toward loudspeaker playbacks of simulated male signals. We relied on a male attractiveness index $(\mathrm{AT}=0.524 \mathrm{PA}+0.296 \mathrm{AI}+0.117 \mathrm{PR})$ derived via the multivariate regression analysis (Lande \& Arnold, 1983) of an earlier generation (fifth) of the stock population, to design the simulated signals used (multivariate regression model 1, see Jang \& Greenfield, 1998). Two different signals, of which the expected attractiveness (AT) in the population at large was equal, but of which specific characters were quite different, were designed and constructed on a personal computer using a digital signal editing program. We constructed one signal with a high $\mathrm{PR}$ value $\left(56.5 \mathrm{~s}^{-1}\right), 2.53 \mathrm{SD}$ above the population mean, and a commensurately short AI value $(180 \mu \mathrm{s}), 1.00 \mathrm{SD}$ below the population mean (Fig. 1). The other signal bore reciprocal characters: low PR $\left(36.5 \mathrm{~s}^{-1} ; 2.53 \mathrm{SD}\right.$ below the mean) but long $\mathrm{AI}(845 \mu \mathrm{s} ; 1.00 \mathrm{SD}$ above the mean). We repeated the same standard pulse, $108 \mu$ s in length and $99 \mathrm{kHz}$ in peak frequency, in both the high-PR and long-AI signals. This standard pulse had been recorded (see below for method) from a male in the stock population and approximated the population means in length and frequency.

All four PR and AI values of test signals fell within the ranges observed in the $\mathrm{P}_{1}$ generation. Moreover, the combinations of the test signals used in this study, and even more extreme combinations, were found in the $\mathrm{P}_{1}$ generation. Based on multivariate regression studies, there is no interaction effect between $\mathrm{AI}$ and $\mathrm{PR}$ on male attractiveness $\left(\gamma_{\mathrm{PR}, \mathrm{AI}}=0.052, P=0.208\right.$; see Jang \& Greenfield, 1998). Because our test signals are based on selection gradient studies on the male a

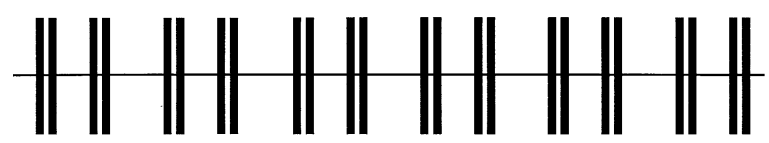

b

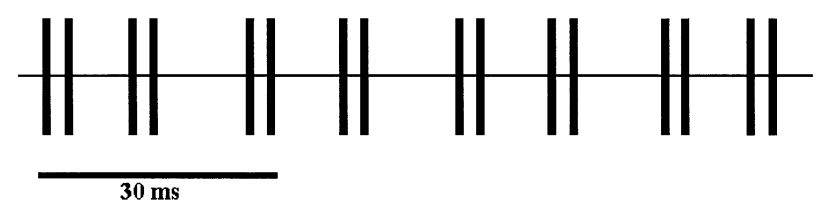

Fig. 1 Male signals used in playback experiments determining female preference indices for Achroia grisella. (a) The highpulse rate signal. This signal had a $56.5-\mathrm{s}^{-1}$ pulse rate $(2.53 \mathrm{SD}$ above the population mean) and a $180-\mu$ s asynchrony interval (1.00 SD below the mean). (b) The long-asynchrony interval signal. This signal had a $36.5-\mathrm{s}^{-1}$ pulse rate $(2.53 \mathrm{SD}$ below the mean) and a $845-\mu$ s asynchrony interval (1.00 SD above the mean). All pulses in both signals were identical in spectral and amplitude properties.

attractiveness index, the effects of different AI and PR values on female preference should be independent of each other.

The two playback signals were continuously and simultaneously played (looped) back using a custom computer program developed specifically for recording and playing back ultrasound. We used a sampling rate of $597 \mathrm{kHz}$ during each trial. The signals were transformed into two analogue outputs in the soundcard (Supersound Engineering Version, SiliconSoft, San Jose, CA) of a personal computer (80486 processor; $66 \mathrm{MHz} \mathrm{cpu}$ ). These outputs were amplified (UltraSound Advice model S55 amplifier; frequency response $\pm 3 \mathrm{~dB}$ from 18 to $300000 \mathrm{~Hz}$ ) and broadcast from ultrasonic loudspeakers (UltraSound Advice model S56; frequency response from 10 to $200000 \mathrm{~Hz}$ ). Before each testing session, we recorded the two signals played back by the two ultrasonic loudspeakers to ensure that the amplitude, frequency and temporal signal characters actually broadcast at the female release point were the intended ones. In all experiments, PA values, recorded as digitized voltages ordered on a linear scale, were adjusted (via amplifier gain controls) and remeasured until broadcasts from the two loudspeakers both equalled $79( \pm 0.3) \mathrm{dB}$ SPL at the female release point.

The two loudspeakers were placed immediately outside the perimeter of a circular screen arena $(80 \mathrm{~cm}$ diameter) and directed towards the arena centre (Fig. 2). Azimuthal separations between the loudspeakers were $120^{\circ}$ relative to the centre. The arena was kept in a $3.0 \times 3.5 \times 2.5-\mathrm{m}$ semi-anechoic room whose photo- 


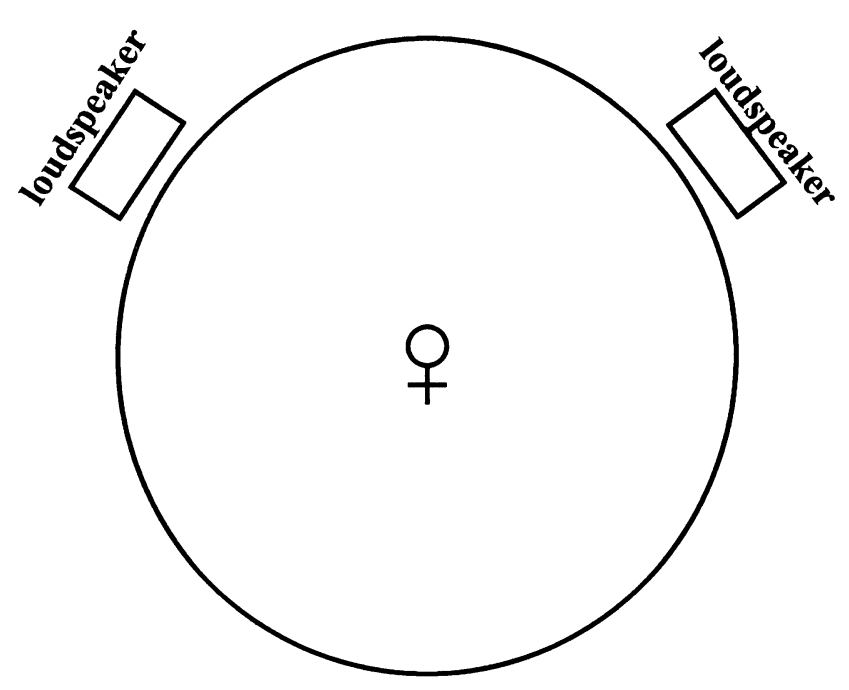

Fig. 2 A schematic diagram for the playback experimental setup. Female Achroia grisella were released from the centre of the circular screen arena ( $80 \mathrm{~cm}$ diameter) and were given a choice of two signals: high-pulse rate and long-asynchrony interval signals. Two ultrasonic loudspeakers were placed immediately outside the perimeter of the area and were azimuthally separated by $120^{\circ}$ relative to the arena centre.

period and temperature were maintained as for rearing of the stock population.

We determined female preference indices by releasing females individually and sequentially from a small cup placed at the arena centre. Releases were not made until each female had rested at the centre for at least $15 \mathrm{~s}$. A female was judged to prefer a playback signal if she moved to within $5 \mathrm{~cm}$ of the loudspeaker broadcasting it. Females that did not respond within 2 min of release were not used in further analyses or breeding. All playback experiments were conducted during the initial half of scotophase, the period of peak mating activity in A. grisella (Greenfield \& Coffelt, 1983).

Newly eclosed adult females, both parental $\left(\mathrm{P}_{1}\right)$ and filial $\left(\mathrm{F}_{1}\right)$ generations of experimental animals, were tested three times daily for two consecutive days beginning 6-12 $\mathrm{h}$ after eclosion. Thus, every female was tested a total of six times. For a given female, consecutive trials were separated by at least $30 \mathrm{~min}$ to avoid potential habituation to playback signals. The loudspeakers broadcasting the two playback signals, 'high-PR' and 'long-AI', were switched each time a given female was tested to eliminate any confounding directional influences on her preference. Our six-trial test yielded seven possible preference index values for each female, ranging from 0 (all responses to the long-AI signal) to 6 (all responses to the high-PR signal).

\section{Experimental pairings and breeding}

We used a half-sib breeding design (see Becker, 1984; Falconer, 1989) to determine heritability of the female preference index. On each of two consecutive days, a given male was paired with a different female whose preference index had been determined. Each pair was placed in a small screen cage, and mating usually ensued regardless of the male's attractiveness. After mating, the male was removed, and deposited eggs were collected. Twenty-six males were each mated to two females, five males were each mated to one female, and one male was mated to three females. Thus, the experimental pairings consisted of 60 full-sib families.

Eggs $\left(F_{1}\right)$ collected from each of the pairings were placed on $30 \mathrm{~g}$ of diet in a $17 \times 12 \times 6.5-\mathrm{cm}$ plastic container kept in the chamber used for rearing the stock population. After hatching and attaining the second instar, 40 larvae were randomly chosen from among the offspring of each mated female $\left(\mathrm{P}_{1}\right)$ and placed individually in $30-\mathrm{mL}$ containers with $1 \mathrm{~g}$ diet. Following adult eclosion, females $\left(F_{1}\right)$ were tested for their preference indices. The same protocols used for measuring female preference indices in the $P_{1}$ generation were used in the $\mathrm{F}_{1}$ generation.

\section{Statistical analyses}

From the half-sib breeding design, we calculated the narrow-sense heritability $\left(h_{\mathrm{s}}^{2}\right.$ ) (see Falconer, 1989) for the female preference index. Because distributions of female preference indices for both generations were normal (see Results), we used a nested analysis of variance (ANOvA, Model II) with unequal sample sizes (Sokal \& Rohlf, 1981, pp. 294-299) to partition phenotypic variance for these indices into components resulting from fathers, mothers and offspring. Heritability was calculated as $4 \times$ the proportion of variance attributed to fathers (Falconer, 1989, p. 170, table 10.4). The standard error for the heritability of the female preference index followed Becker (1984, p. 59). To provide an additional estimate of evolvability, we also calculated the coefficient of additive genetic variance, $C V_{\mathrm{A}}=\sqrt{ } V_{\mathrm{A}} /$ $\bar{x}$, where $\bar{x}$ is the mean value of the trait (preference index) (Houle, 1992).

\section{Results}

The distribution of preference indices in the $F_{1}$ generation, averaged for each family, was normal (AndersonDarling normality test, $P=0.199$ ). A ln transformation served to normalize the distribution of preference indices in the $\mathrm{P}_{1}$ generation (Anderson-Darling normality test, $P=0.721)$. The means of mothers' $(3.43 \pm 1.57 \mathrm{SD}$,

(C) The Genetical Society of Great Britain, Heredity, 84, 73-80. 
$N=60)$ and daughters' $(3.40 \pm 0.605 \mathrm{SD}, \quad N=60)$ preference indices did not differ significantly (Wilcoxon two-sample test, $W=3688, P=0.76$ ).

The means of preference indices for both generations deviated from 3.00, the expected value assuming equivalent attractiveness of the two simulated signals. Instead, the proportion of all 900 orientations ( $N=150$ females, six trials per female) towards the high-PR signal in the $\mathrm{P}_{1}$ generation was 0.593 , and the proportion of all 3024 trials in the $F_{1}$ generation $(N=504$ females, six trials per female) toward the high-PR signal was 0.573 . These observed proportions were significantly different $(P<0.0001$ for both generations; binomial test with normal approximation) from the expectation that equal numbers of responses should be directed towards each simulated signal. This discrepancy may reflect a change in female preference indices over the 11 generations elapsing between determination of the male attractiveness index and the study reported here. Estimation of heritability relies on consistent measurements over two successive generations $\left(\mathrm{P}_{1}\right.$ and $F_{1}$ ) rather than on values of the preference indices themselves. Because means of preference indices for both $P_{1}$ and $F_{1}$ generations were statistically similar, our method of quantification of female preference was consistent for both generations. Therefore, the observed departure from the initial expectation of equivalent orientation towards both test signals would not have affected our interpretation of the results.

We used the observed proportions of orientation towards the high-PR signal, 0.593 and 0.573 , for the $\mathrm{P}_{1}$ and $F_{1}$ generations, respectively, to calculate the expected binomial frequency distributions of female preference indices. In the six-trial tests, $31 \%$ of mothers and $23 \%$ of daughters preferred the high-PR signal five or six times, and $12 \%$ of mothers and $8 \%$ of daughters preferred the long-AI signal five or six times (Fig. 3). The observed frequency distributions of female preference indices were significantly different from the expected ones (binomial goodness-of-fit tests; for $\mathrm{P}_{1}$ : $\chi_{4}^{2}=45.5, P<0.0001$; for $\mathrm{F}_{1}: \chi_{4}^{2}=20.1, P=0.0002$ ), indicating that more females than expected by chance always chose the high-PR or the long-AI signal.

ANOVA of the female preference index among the $F_{1}$ generation (daughters distributed among half-sib and full-sib families) revealed a significant added variance component attributable to fathers (Table 1). The heritability of the female preference index, estimated from ANOvA, was marginally significant $\left(h_{\mathrm{s}}^{2}=0.212\right.$, $\mathrm{SE}=0.1347, P=0.0611)$. The $C V_{\mathrm{A}}$ of the female preference index was 0.1824 . We also averaged the preference indices of daughters in each full-sib family and regressed these averages against the mother's index to obtain a second heritability estimate. The correlation of

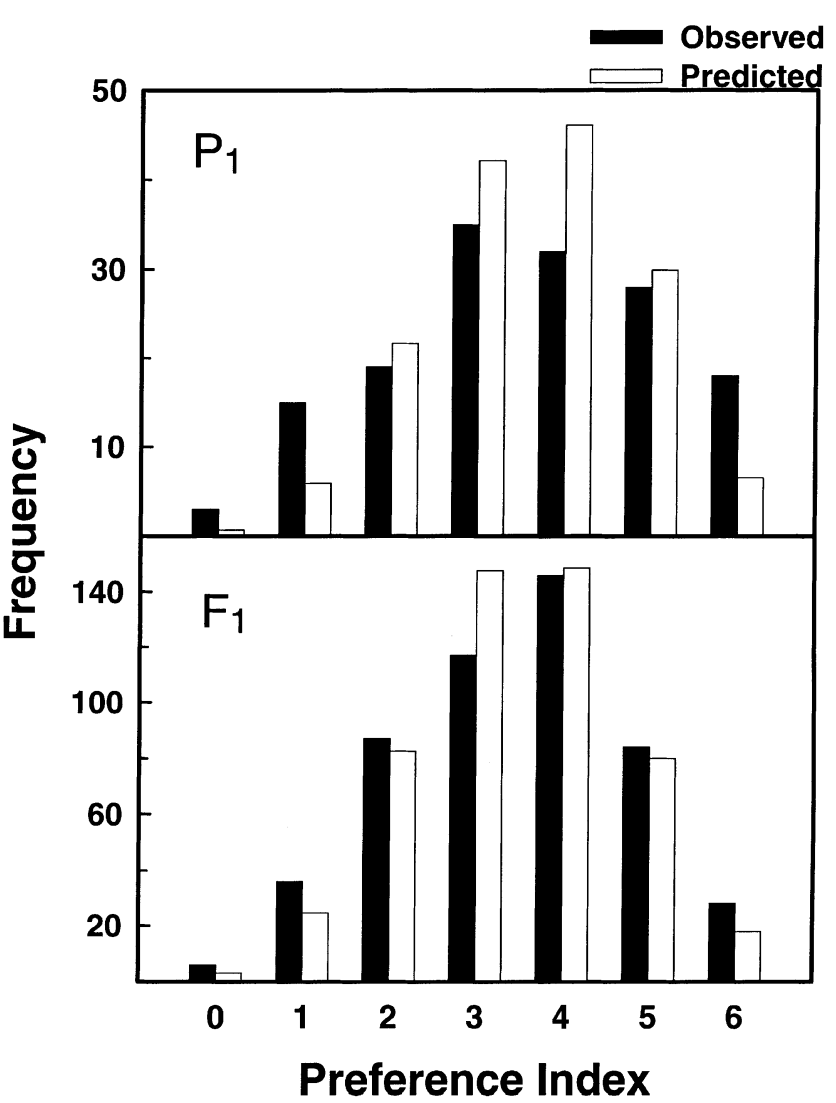

Fig. 3 Frequency histograms of mothers' $\left(\mathrm{P}_{1}\right)$ and daughters' $\left(\mathrm{F}_{1}\right)$ preference indices. Female Achroia grisella $\left(\mathrm{P}_{1}, N=150\right)$ were presented with a choice of a high-PR (pulse rate) signal and a long-AI (asynchrony interval) signal, which were played back from loudspeakers. The preference index is the number of times that a female chose the high-PR signal in six repeated trials. After testing, each of two females $\left(\mathrm{P}_{1}\right)$ were mated with a male randomly chosen from a population. The preference indices of female offspring $\left(\mathrm{F}_{1}, N=504\right)$ were assessed according to the same methods as used for the mothers $\left(\mathrm{P}_{1}\right)$. Predicted values are based on binomial frequency distributions calculated using mean proportions 0.593 and 0.573 for the $\mathrm{P}_{1}$ and $F_{1}$ generations, respectively.

preference index between mothers and their daughters was positive and significant (least squares linear regression weighted by the number of daughters; $b_{\mathrm{F} 1, \mathrm{P} 1}=0.119, t=2.89, P=0.005$; Fig. 4). Of course, this correlation may result, in part, from maternal effects (Falconer, 1989).

\section{Discussion}

Assessment of phonotactic choices in playback experiments showed that female preference indices varied among individuals in A. grisella. Such phenotypic variance of female preference within a population is 
Table 1 Analysis of variance (ANOVA) table illustrating variation in female preference index in Achroia grisella. After recording signals, each male $\left(\mathrm{P}_{1}\right)$ was usually mated with two females whose preference indices had been determined in six-trial choice experiments. Because of this half-sib design, the 'mothers' in the source column were nested within 'fathers' for analyses. From each full-sib family $\left(F_{1}\right)$, signals were recorded from a sample of 5-10 of the male offspring, and preference indices were assessed from a sample of 4-14 of the female offspring

\begin{tabular}{lrrrc}
\hline Source & d.f. & \multicolumn{1}{c}{ SS } & $F$ & $P$ \\
\hline Fathers & 31 & 99.38 & 2.01 & $<0.01$ \\
Mothers & 28 & 44.75 & 0.93 & $>0.4$ \\
Offspring & 441 & 760.73 & & \\
\hline
\end{tabular}

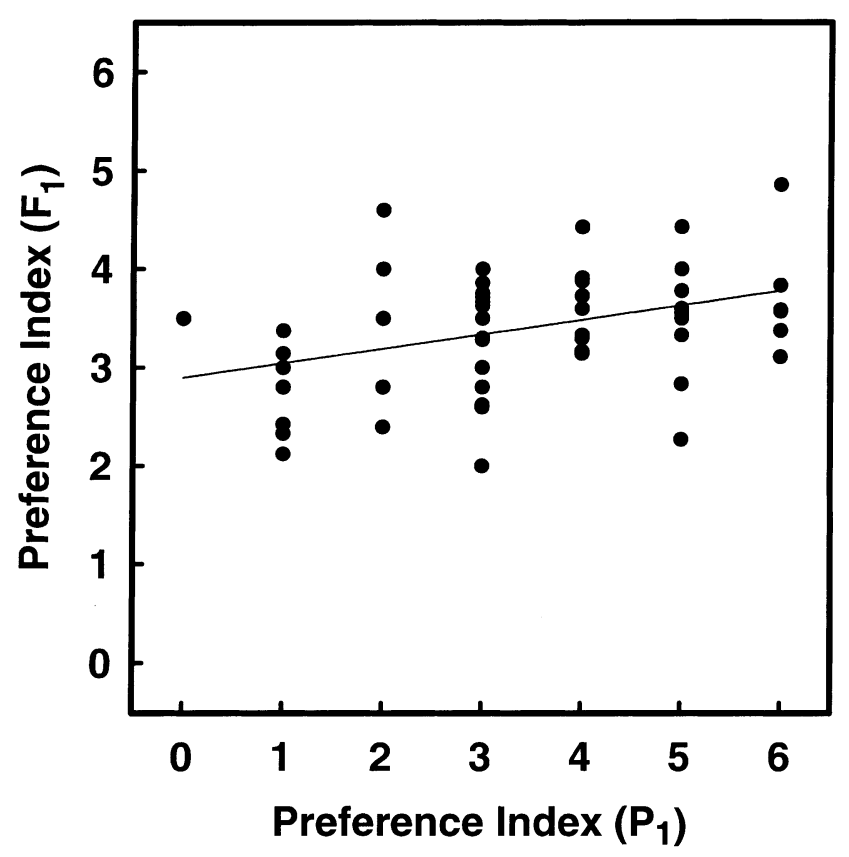

Fig. 4 Regression of daughters' preference indices on their mother's preference index in Achroia grisella. The preference indices of female offspring of each full-sib family were averaged and regressed against their mother's preference index (weighted by the number of daughters).

documented in the sulphur butterfly (Colias eurytheme; Sappington \& Taylor, 1990), the field cricket (Gryllus integer; Wagner et al., 1995), the cricket frog (Acris crepitans; Ryan et al., 1992), the barn swallow (Hirundo rustica; Møller, 1994) and the guppy (Poecilia reticulata; Godin \& Dugatkin, 1995). Although phenotypic variance of female preference is a prerequisite for selection to operate on the preference, the phenotypic variance does not necessarily imply additive genetic variance (heritability) of female preference. Indirect models of sexual selection assume additive genetic variances for both female preference and preferred male traits, and these variances are usually examined by quantitative genetic experiments.

The significant variation in preference indices included consistent responses towards high-PR or long-AI signals in some females (Fig. 3). Although three signal characters, including PR and AI, are the direct targets of female choice at a population level, our findings suggest that individual females differ in the relative importance of various signal characters during mate assessment. ANOvA of data from our half-sib breeding design indicates that additive genetic variation accounts for a substantial portion of this variation in female preference.

The heritability and evolvability estimates of the female preference index imply that this trait can respond to various selection pressures. Potential direct selection pressures include energetic costs and risks incurred while searching for mates. Alternatively, or additionally, selection may act indirectly on the female preference trait via male signal traits genetically correlated with female preference. Under such circumstances, a correlated response of female preference to selection on the male trait may result. Such correlated responses form the core of indirect sexual selection mechanisms, including arbitrary and good-genes selection (Kirkpatrick \& Ryan, 1991). The existence of heritable variation in both male signal characters (Collins et al., 1999) and female preference in A. grisella suggests that an indirect mechanism of sexual selection can operate (see Jia \& Greenfield, 1997). An earlier attempt to detect any genetic correlation between the female preference index and male signal characters failed, and this absence of the genetic correlation probably reflects the lack of assortative mating during the many generations of laboratory breeding prior to quantitative genetic experiments (Jang, 1997; see also Bakker \& Pomiankowski, 1995). Obviously, further work investigating genetic correlations between male signal and female preference traits in field populations and field studies of the mating pattern in natural population will be necessary to understand more fully the nature of sexual selection in A. grisella.

Our experimental design not only allowed us to estimate the evolvability of female preference but also revealed the manner in which this trait may vary within a population. Although female preference has been measured quantitatively in several taxa, these measurements entailed testing responses to a range of values from the one-dimensional distribution of a male trait [e.g. the three-spined sticklebacks (Gasterosteus aculeatus), Bakker, 1993)]. Instead, we selected our test signals 
from among the multidimensional coordinates of male signal characters such that their individual characters differed markedly but their expected attractiveness was equivalent. This careful design of test signals was implemented to maximize the chance of detecting individual variation in female preference for the multidimensional signal.

Female preference may be studied via bioassaying response to single-stimulus presentations or two-stimulus choice experiments. Although single-stimulus experiments provide information that may be used to construct readily a precise preference function (e.g. Ritchie, 1996; Wagner, 1998), multiple-stimulus designs may offer the opportunity to detect the relative attractiveness of the various stimuli, differences that may not be evident from single-stimulus presentations (Doherty, 1985). We chose to rely on the two-stimulus design to take advantage of this acute resolving power and because females in natural populations are generally confronted with a simultaneous choice of two or more signalling males. Studies of indirect sexual selection remain hindered by difficulties in quantifying the female preference trait. Our study shows that careful selection of test signals and an experimental design tailored for the behaviour of the experimental species will greatly enhance determination of individual variation of this critical yet elusive trait.

\section{Acknowledgements}

We are indebted to David Alexander, Butch Brodie, Feng-you Jia, Klaus Reinhold, Gunther Schlager, Larry Sherr, Norm Slade, Andy Snedden and Jason Wolf for helpful discussions and critical comments on this manuscript. Robert Minckley collected the moths used to found our stock population, and June Newman, Thomas Weber and Kelly Williams provided indispensable laboratory assistance with rearing and testing of the moths. This study was supported financially by U.S. National Science Foundation grant IBN 94-07304.

\section{References}

BAKKeR, T. C. M. 1993. Positive genetic correlation between female preference and preferred male ornament in sticklebacks. Nature, 363, 255-257.

BAKKER, T. C. M. AND POMIANKOWSKI, A. 1995. The genetic basis of female mate preferences. J. Evol. Biol., 8, 129-171.

BECKER, W. A. 1984. Manual of Quantitative Genetics. Academic Enterprises, Pullman, WA.

COLLINS, R. D., JANG, Y., REINHOLD, K. AND GREENFIELD, M. D. 1999. Quantitative genetics of ultrasonic advertisement signalling in the lesser waxmoth, Achroia grisella (Lepidoptera: Pyralidae). Heredity, 83, 644-651.

DOHERTy, J. A. 1985. Phonotaxis in the cricket, Gryllus bimaculatus De Geer: Comparisons of choice and no-choice paradigms. J. Comp. Physiol. A, 157, 279-289.

DUTKY, S. R., THOMPSON, J. V. AND CANTWELL, G. E. 1962. A technique for mass rearing the greater wax moth. Proc. Ent. Soc. Wash., 64, 56-58.

FALCONER, D. S. 1989. Introduction to Quantitative Genetics, 3rd edn. John Wiley, New York, NY.

GODIN, J.-G. J. AND DUGATKIN, L. A. 1995. Variability and repeatability of female mating preference in the guppy. Anim. Behav., 49, 1427-1433.

GREENFIELD, M. D. AND COFFELT, J. A. 1983. Reproductive behaviour of the lesser waxmoth, Achroia grisella (Pyralidae: Galleriinae): Signalling, pair formation, male interactions, and mate guarding. Behaviour, 84, 287-315.

HOULE, D. 1992. Comparing evolvability and variability of quantitative traits. Genetics, 130, 195-204.

JANG, Y. 1997. Evolution and Genetics of Mate Preference in an Ultrasonic Pyralid Moth. PhD Dissertation, University of Kansas.

JANG, Y. AND GREENFIELD, M. D. 1996. Ultrasonic communication and sexual selection in wax moths: female choice based on energy and asynchrony of male signals. Anim. Behav., 51, 1095-1106.

JANG, Y. AND GREENFIELD, M. D. 1998. Absolute versus relative measurements of sexual selection: assessing the contributions of ultrasonic signal characters to mate attraction in lesser wax moths, Achroia grisella (Lepidoptera, Pyralidae). Evolution, 52, 1383-1393.

JANG, Y., COLLINS, R. D. AND GREENFIELD, M. D. 1997. Variation and repeatability of ultrasonic sexual advertisement signals in Achroia grisella (Lepidoptera: Pyralidae). J. Insect Behav., 10, 87-98.

JENNIONS, M. D. AND PETRIE, M. 1997. Variation in mate choice and mating preferences: a review of causes and consequences. Biol. Rev., 72, 283-327.

JIA, F.-Y. AND GREENFIELD, M. D. 1997. When are good genes good? Variable outcomes of female choice in wax moths. Proc. R. Soc. B, 264, 1057-1063.

KIRKPATRICK, M. 1982. Sexual selection and the evolution of female choice. Evolution, 36, 1-12.

KIRKPATRICK, M. AND RYAN, M. J. 1991. The evolution of mating preferences and the paradox of the lek. Nature, 350, $33-38$.

LANDE, R. 1981. Models of speciation by sexual selection on polygenic traits. Proc. Natl. Acad. Sci. U.S.A., 78, 3721-3725.

LANDE, R. AND ARNOLD, S. J. 1983. The measurement of selection on correlated characters. Evolution, 37, 1210-1226.

MøLLER, A. P. 1994. Repeatability of female choice in a monogamous swallow. Anim. Behav., 47, 643-648.

POMIANKOWSKI, A. 1988. The evolution of female mate preferences for male genetic quality. Oxford Surveys in Evolutionary Biology, 5, 137-183.

RITCHIE, M. G. 1996. The shape of female mating preferences. Proc. Natl. Acad. Sci. U.S.A., 93, 14628-14631. 
ROSENQVIST, G. AND BERGLUND, A. 1992. Is female sexual behavior a neglected topic? Trends Ecol. Evol., 7, 174-176.

RYAN, M. J., PERRILL, S. A. AND WILCZYNSKI, w. 1992. Auditory tuning and call frequency predict population-based mating preferences in the cricket frog, Acris crepitans. Am. Nat., 139, 1370-1383.

SAPPINGTON, T. W. AND TAYLOR, O. R. 1990. Disruptive sexual selection in Colias erytheme butterflies. Proc. Natl. Acad. Sci. U.S.A., 87, 6132-6135.
SOKAL, R. R. AND ROHLF, F. J. 1981. Biometry, 2nd edn. W. H. Freeman, New York.

SPANGLER, H. G., GREENFIELD, M. D. AND TAKESSIAN, A. 1984. Ultrasonic mate calling in the lesser wax moth. Physiol. Entomol., 9, 87-95.

WAGNER, W. E. JR 1998. Measuring female mating preferences. Anim. Behav., 55, 1029-1042.

WAGNeR, W. E. JR, MURRAY, A. M. AND CADE, W. H. 1995. Phenotypic variation in the mating preferences of female field crickets, Gryllus integer. Anim. Behav., 49, 1269-1281. 